\title{
Embedded piezoelectrics for sensing and energy harvesting in total knee replacement units
}

\author{
Brooke E. Wilson $^{\mathrm{a}}$, R. Michael Meneghini ${ }^{\mathrm{b}}$, Steven R. Anton*a \\ ${ }^{a}$ Tennessee Technological University, Department of Mechanical Engineering, 115 W. 10th Street, \\ Cookeville, TN, USA 38505-0001 \\ ${ }^{\mathrm{b}}$ Indiana University School of Medicine, Department of Orthopaedic Surgery, 1120 W. Michigan St, \\ Suite 600, Indianapolis, IN USA 46202-5111
}

\begin{abstract}
The knee replacement is the second most common orthopedic surgical intervention in the United States, but currently only 1 in 5 knee replacement patients are satisfied with their level of pain reduction one year after surgery. It is imperative to make the process of knee replacement surgery more objective by developing a data driven approach to ligamentous balance, which increases implant life. In this work, piezoelectric materials are considered for both sensing and energy harvesting applications in total knee replacement implants. This work aims to embed piezoelectric material in the polyethylene bearing of a knee replacement unit to act as self-powered sensors that will aid in the alignment and balance of the knee replacement by providing intraoperative feedback to the surgeon. Postoperatively, the piezoelectric sensors can monitor the structural health of the implant in order to perceive potential problems before they become bothersome to the patient. Specifically, this work will present on the use of finite element modeling coupled with uniaxial compression testing to prove that piezoelectric stacks can be utilized to harvest sufficient energy to power sensors needed for this application.
\end{abstract}

Keywords: total knee replacement, piezoelectric sensing, energy harvesting, finite element method, compression testing

\section{INTRODUCTION}

The practice of using sensors for intraoperative and postoperative sensing has become a subject of growing interest in the field of biomedical research in recent years, particularly in the field of orthopedics. Intraoperative sensors are currently used in total knee replacements (TKR), but they must be removed after the data is collected during surgery ${ }^{1-3}$. There are some in-vivo sensors that use electromagnets as their power source. It is possible for these sensors to remain in place postoperatively, however in order to power these sensors and collect data, a fixture must be attached to the outside of the knee. The use of this external fixturing can impede natural movement and lead to data sets that are not representative of uninhibited human motion ${ }^{4,5}$. The optimum data collection method would allow for collection during surgery as well as during typical daily activities without disturbing the patient.

For reference, Figure 1 provides an X-ray image of a standard total knee replacement showing the femoral component, tibial tray, and location of polyethylene bearing. In a study conducted by Kaufman et al. ${ }^{1}$, a transducer was designed to fit within a standard tibial tray with an overall increase in height of $6 \mathrm{~mm}$ from the standard implant design. The tibial component was instrumented with four wired load cells. These load cells provided data containing the magnitude and location of the forces applied to the load cell. Due to size constraints, the device was unable to measure shear forces generated in the knee. The impetus behind this study was the collection of in-vitro data concerning the most common failure mode of a TKR: lift-off of the tibia's lateral aspect. The work of Morris et al. ${ }^{2}$ was closely related to the study conducted by Kaufman with a few notable differences. The tibial tray used in the study conducted by Morris was instrumented with four load cells, which were placed in the four quadrants of the tibial tray. These load cells transmitted their data wirelessly with a micro transmitter, which was incorporated into the tibial prosthesis. The micro transmitter is then powered using an external coil. Both of these devices have been used in in-vitro testing, but neither has continued on to the in-vivo testing stage ${ }^{1,2}$.

*santon@tntech.edu; phone 1931 372-3287, fax 1931 372-6340

Active and Passive Smart Structures and Integrated Systems 2015, edited by Wei-Hsin Liao,

Gyuhae Park, Alper Erturk, Proc. of SPIE Vol. 9431, 94311E · (c) 2015 SPIE

CCC code: $0277-786 \mathrm{X} / 15 / \$ 18 \cdot$ doi: $10.1117 / 12.2087441$

Proc. of SPIE Vol. $943194311 \mathrm{E}-1$ 


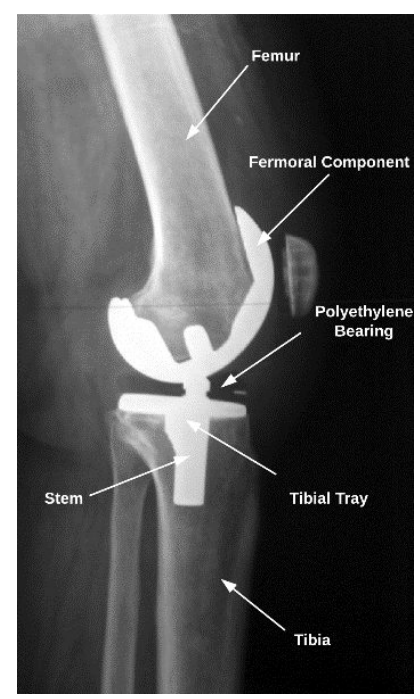

Figure 1. Standard components of a total knee replacement unit

In the work of D'Lima et al. ${ }^{4}$, a standard tibial tray was fitted with a micro transmitter and strain gauges. This instrumented tibial tray was then implanted into a single patient. After three months it was used to collect data while the patient performed normal tasks associated with daily life. This data aligned closely with mathematical models, which have been developed for knee joint forces. While very useful, this data cannot accurately forecast joint loads during unpredictable activities. Kirking et al. ${ }^{5}$ conducted a study using twelve strain gauges, which were installed in the stem of the implant, to measure all six components of the tibial forces. The data was transmitted using a short radio antenna for high frequency transmission from the implant. The devices in both Kirking and D'Lima's work were powered with external electromagnetic coils.

This paper aims to show, through the use of finite element analysis and uniaxial compression testing, that the electricity generated by compressing a piezoelectric element inserted into a knee replacement would be sufficient to power a low power sensor. Specifically, this work aims to embed piezoelectric materials in the polyethylene tibial component of a knee replacement unit to act as sensors that will aid in the alignment and balance of the knee replacement by providing intraoperative feedback to the surgeon. Recently, clinical studies have shown that proper alignment and balance of the implant's ligamentous forces between the medial and lateral compartments during surgery have a significant effect on the success of the operation ${ }^{6,7}$. The piezoelectric sensors can postoperatively monitor the structural health of the implant to detect potential problems before they become bothersome to the patient. It is possible to continually monitor the implant for the duration of its use through multifunctional applications of the piezoelectric devices to also harvest mechanical energy to power the sensor, thus eliminating the need for batteries that are incapable of lasting for the life of the implant.

\section{METHODOLOGY}

\subsection{OpenSim modeling}

It is necessary to profile the force applied to the knee joint by a normal walking gait to accurately predict the voltage and power output of the piezoelectric material embedded in the tibial tray. The development of such a force profile has been the subject of many studies in recent years ${ }^{8-11}$. While these studies have provided valuable information about the force model of the knee joint, none of these models have produced a predictive model that closely matches data collected from instrumented knees.

A force profile (shown later in Figure 5) was developed using OpenSim ${ }^{12}$, which is an open source biomechanical modeling software that was developed at Stanford University intended for modeling of musculoskeletal structures and simulation of dynamic movement ${ }^{13}$. To calculate joint reaction loads, it is first necessary to set up a relevant model and static optimization. This paper utilizes a model file, which is included in the basic OpenSim software package. This 


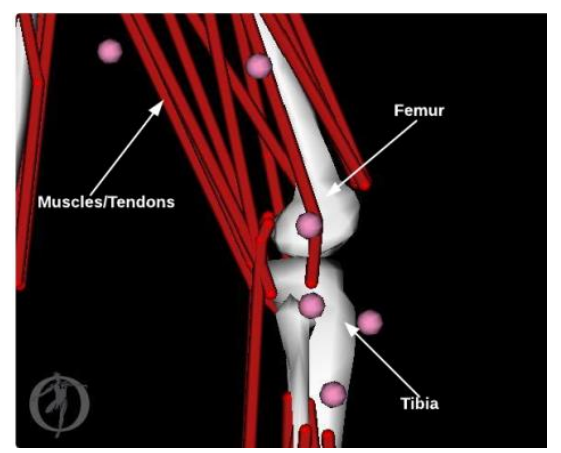

Figure 2. Geometry used in OpenSim modeling.

model is a 165.7 pound male who is 70.8 inches tall. The knee joint of this model is shown for reference in Figure 2. This model is then used to create a static optimization study. The static optimization study utilizes many different inputs: such as motion files, force actuators, and external load, which makes OpenSim modeling exceedingly customizable. The parameters used to establish the static optimization study can then be ported directly into the joint reaction analysis tool. Once the joint reaction analysis tool has been used, the forces calculated can be exported to be used in further calculations. MATLAB is used in this study for further analysis of these joint reaction loads.

\subsection{Electromechanical modeling}

A key feature of piezoelectric material is the ability to convert mechanical strain energy to electrical output. This electromechanical coupling can be shown by the piezoelectric constitutive relations, given in compact (Voight) notation ${ }^{14}$ as

$$
\begin{aligned}
& S_{i}=s_{i j}^{E} T_{j}+d_{i k} E_{k} \\
& D_{m}=d_{m j} T_{j}+\varepsilon_{m k}^{T} E_{k}
\end{aligned}
$$

wherein $S$ represents mechanical strain, $s^{E}$ represents the elastic compliance, $T$ represents the mechanical stress, $d$ represents the piezoelectric strain constant, $E$ represents the electrical field, $D$ represents the electrical displacement, and $\varepsilon^{T}$ represents the dielectric constant.

In this work, a piezoelectric stack is placed under compressive loading from the knee forces calculated using OpenSim. The ' 33 ' operating mode of the piezoelectric is utilized in this configuration, and the only nonzero stress and electric field are in the ' 3 ' direction, leaving

$$
\begin{aligned}
& S_{3}=s_{3}^{E} T_{3}+d_{33} E_{3} \\
& D_{3}=d_{33} T_{3}+\varepsilon_{33}^{T} E_{3}
\end{aligned} .
$$

If the electrodes of each piezoelectric layer are connected in parallel to a single resistive load, then the governing equation can be obtained by applying Gauss's law as follows

$$
\sum_{i=1}^{N} \frac{d}{d t}\left(\int D \cdot n d A\right)=\frac{v(t)}{R}
$$

where $N$ is the number of layers in the stack, $A$ is the surface area of the electrode (it is assumed that the electrode covers the entire piezoelectric surface), $D$ is the vector of electric displacement, $n$ is the vector of outward normal of the electrode, and $v(t)$ is the voltage output across the load resistor, $R$. Applying Eq.(3) to the $D_{3}$ expression given in Eq.(2) and noting that stress can be expressed as $T_{3}=F(t) / A$, where $F(t)$ is the compressive force applied to the 
piezoelectric stack, and the electric field can be stated as $E_{3}=-v(t) / h$, where $h$ is the thickness of each piezoelectric layer, gives the governing equation of the system as

$$
C_{p}^{e f f} \frac{d v(t)}{d t}+\frac{v(t)}{R}=d_{33}^{e f f} \dot{F}(t)
$$

where $C_{p}^{e f f}$ is the effective piezoelectric capacitance and $d_{33}^{\text {eff }}$ is the effective piezoelectric constant. The effective piezoelectric capacitance can be expressed as

$$
C_{p}^{e f f}=\frac{N \varepsilon_{33}^{T} A}{h}
$$

and the effective piezoelectric constant can be expressed as

$$
d_{33}^{e f f}=N d_{33} .
$$

$P$, the instantaneous power, can be calculated with the expression

$$
P(t)=\frac{v^{2}(t)}{R}
$$

where the average power, $P_{a v g}$, can be found by integrating the instantaneous power as follows

$$
P_{\text {avg }}=\frac{1}{T} \int_{0}^{T} \frac{v^{2}(t)}{R} d t
$$

where $T$ is the time used in the simulation.

It should be noted that the governing expression given in Eq.(4) is a first order expression for the voltage generated by a piezoelectric cylinder due to an input force. This expression, unlike typical resonant energy harvester models, is only valid for excitation frequencies that fall far below the resonance of the cylinder, where the device exhibits first-order dynamics. Therefore, the frequency of the input force must remain low in relation to the stack resonance.

\subsection{Finite element modeling}

To predict the voltage output without having to build the correlated physical system with many different parameters, it is advantageous to use finite element modeling to predict the behavior of the system being studied. In this study, ANSYS has been utilized to create a finite element model of the polyethylene TKR bearing with embedded piezoelectric, which has 25,419 nodes and 10,643 elements, as seen in Figure 3, using SOLID186 and SOLID 187 elements. Once the

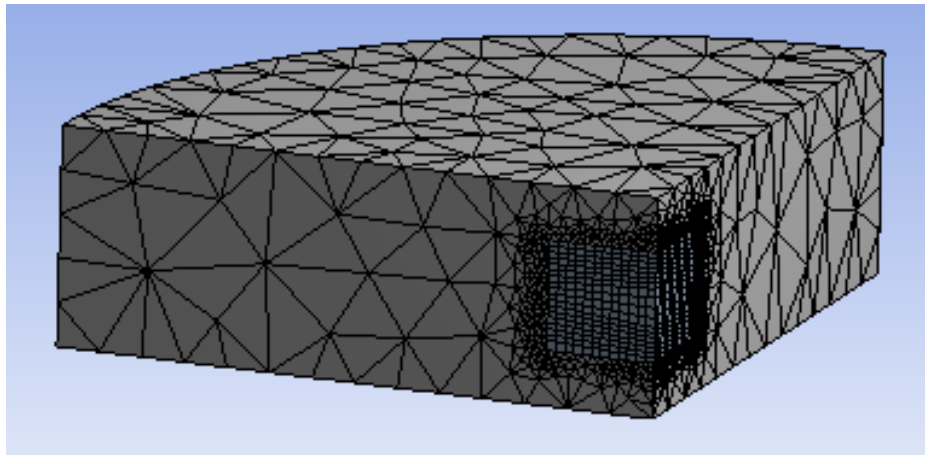

Figure 3. ANSYS mesh. 
percentage of force transferred through the bearing to the piezoelectric device is determined, the force profile is then run through the MATLAB architecture as seen in the author's previous work ${ }^{12}$ to determine the voltage output of the embedded piezoelectric device. The system has been simplified from the complex geometry found in a TKR bearing to a simple cylindrical geometry so that the finite element model can be verified with simple uniaxial compression testing. It should be noted that the eventual design will incorporate four piezoelectric transducers (one in each of the anterolateral, anteromedial, posterolateral, and posteromedial compartments of the knee), however, a single embedded piezoelectric is investigated in this study for simplicity. The cylinder is modeled as ultra high molecular weight polyethylene $(\mathrm{UHMW})^{15}$, and has been modeled having a thickness ranging from $5 \mathrm{~mm}$ to $10 \mathrm{~mm}$ in $1 \mathrm{~mm}$ increments. $5 \mathrm{~mm}$ to 10 $\mathrm{mm}$ is considered a realistic range of thicknesses for UHMW TKR bearings given current TKR implant designs. Each cylinder is initially modeled as having a monolithic piezoelectric element embedded in the center. While piezoelectric stacks are of interest, a monolithic piezoelectric is initially investigated due to commercial availability for the prototype discussed in the following section. For each thickness of UHMW investigated, a piezoelectric element of varying thicknesses is included from a one millimeter minimum to a maximum of the thickness of the UHMW insert minus two millimeters, as seen in Table 1, to allow for a sufficiently thick layer of UHMW on either side of the piezoelectric.

Table 1. ANSYS testing parameters.

\begin{tabular}{ccccccc} 
& \multicolumn{7}{c}{ UHMW Bearing Thickness (mm) } \\
\cline { 2 - 7 } & $\mathbf{5}$ & $\mathbf{6}$ & $\mathbf{7}$ & $\mathbf{8}$ & $\mathbf{9}$ & $\mathbf{1 0}$ \\
\cline { 2 - 7 } & 1 & 1 & 1 & 1 & 1 & 1 \\
& 2 & 2 & 2 & 2 & 2 & 2 \\
Piezoelectric & 3 & 3 & 3 & 3 & 3 & 3 \\
Element & & 4 & 4 & 4 & 4 & 4 \\
Thickness (mm) & & 5 & 5 & 5 & 5 \\
& & & & 6 & 6 & 6 \\
& & & & & 7 & 7 \\
\end{tabular}

(a)

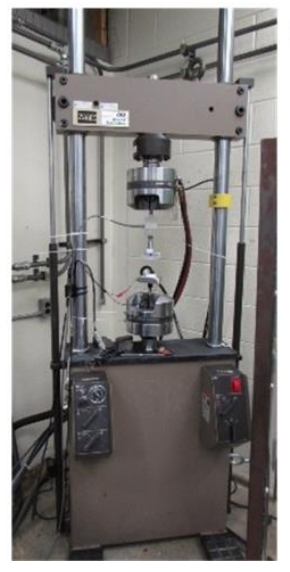

(b)

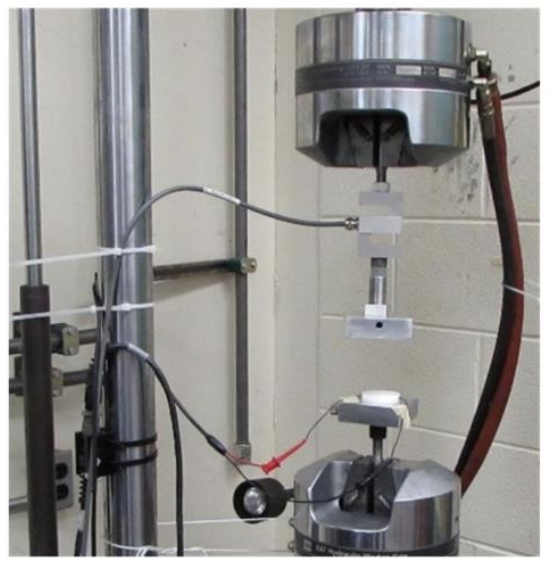

(c)

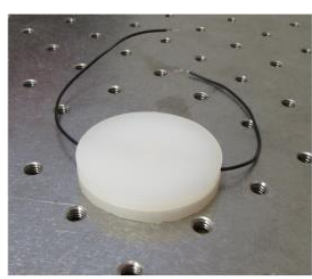

(d)

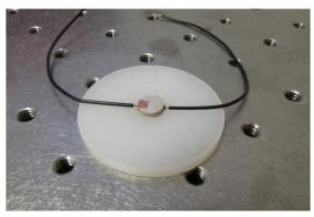

Figure 4. Experimental compression test setup including (a) MTS 810 servo hydraulic load frame, (b) close-up view of compression fixturing, (c) assembled prototype bearing, and (d) prototype bearing showing inserted piezoelectric. 


\subsection{Uniaxial compression testing}

To verify the models created in both ANSYS and MATLAB it is necessary to perform physical experimentation. Uniaxial compression testing is conducted using an MTS 810, Model 647.10A-01 servo-hydraulic load frame, which can be seen in Figure 4(a)-(b). Due to mechanical constraints of the machine it is impossible to replicate the desired load profile with complete accuracy, therefore some approximations are necessary. The specimen used in the uniaxial compression tests is an $8 \mathrm{~mm}$ thick disk of UHMW, which is cut into two $4 \mathrm{~mm}$ thick halves with a $1.5 \mathrm{~mm}$ deep pocket milled in each half with an $8 \mathrm{~mm}$ by $3 \mathrm{~mm}$ APC 850 (PZT-5A) monolithic piezoelectric cylinder embedded in the center. As mentioned above, monolithic piezoceramics are used in the prototype device due to availability, however, stack configurations will be investigated numerically once the models are validated. Tests were run in order to determine the voltage output across a series of resistances, ranging from $100 \mathrm{k} \Omega$ to $1 \mathrm{M} \Omega$, from the monolithic piezoelectric cylinder installed directly into the load frame fixture (with no UHMW bearing), and from the piezoelectric cylinder embedded in the disk of UHMW, both subjected to the load profile generated using OpenSim and shown in Figure 5. It should be noted that the load profile corresponds to a quarter of the tibiofemoral force calculated in OpenSim since the prototype contains a single piezoelectric device and the eventual instrumented implant will contain four embedded piezoelectrics. The voltage output data is acquired during compression testing using a National Instruments NI-9215 data acquisition card, and the applied force is recorded directly in the MTS load frame software.

\section{RESULTS \& DISCUSSION}

The OpenSim joint reaction load simulation discussed earlier ${ }^{12}$ generates the force profile (a quarter of the total tibiofemoral force) that can be seen in Figure 5. This force profile has two distinct peaks. The first peak correlates to the impact force from the heel striking the ground; the second peak correlates to the maximum force experienced by the knee joint as weight is transferred from one leg to the other. In D'Lima et al. ${ }^{16}$, peak forces are given as 2.5-2.8 times body weight for a normal walking gait. These numbers correlate closely to the force profile generated with OpenSim.

The force profile generated in OpenSim is used as the input for the MTS load frame controller software. A comparison between the desired load profile and the load profile generated by the load frame can be seen in Figure 5. While there are some discrepancies, the generated load profile tracks the desired profile well. The data collected from uniaxial compression testing is used to verify that the models constructed in ANSYS and MATLAB generate expected results. A comparison of the generated voltage and the simulated voltage output for a monolithic piezoelectric alone and a monolithic piezoelectric embedded in a disk of UHMW can be seen in Figure 6 and Figure 7, respectively.

It can be seen from the comparison of the data collected in uniaxial compression testing and the simulated data from the models that the model results match the experimental results with reasonable accuracy. While very similar, the results, however, are not entirely identical. The differences between the results can be partially attributed to the nonlinear behavior of $\mathrm{UHMW}^{17}$ and partially attributed to geometric simplifications made in the finite element analysis. It can also be seen that the data collected has many small peaks and valleys that do not appear in the simulation results. This can be attributed to the piezoelectric cylinder discharging slightly as the load varies. The magnitude of the voltage generated

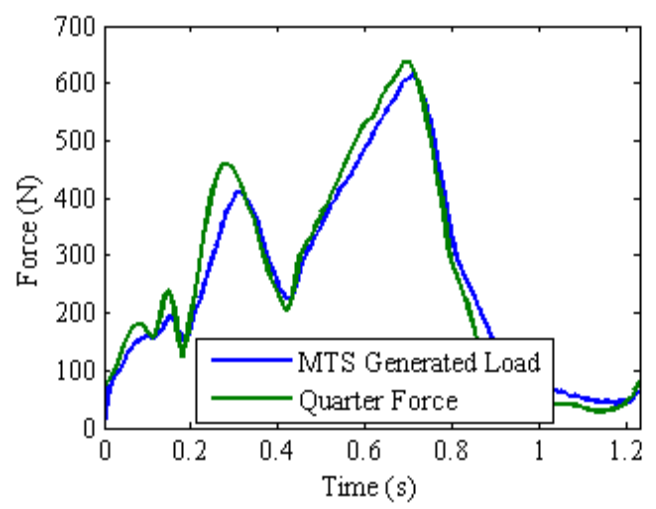

Figure 5. Comparison of the load profile generated by OpenSim with the profile generated by the MTS test frame. 
displays slight discrepancies which may be attributed to the assumed boundary conditions used in ANSYS. Overall, the model results compare favorably to the experimental results, thereby validating the modeling framework adopted in this research.

Now that the model has been verified it can be used to determine the maximum achievable power output given the space constraints of the system. As in the author's previous work ${ }^{12}$ a piezoelectric stack will be used in order to maximize average power in such a small space. The parametric study was conducted using a PZT-5H stack with 100 layers as it was previously found to give the best power output for its cost in comparison to PZT-4, PZT-5A, PZT-8, and PMN-PT ${ }^{12}$. The results of this parametric study can be seen in Figure 8. Again, the overall UHMW thickness as well as the thickness of the embedded piezoelectric devices are varied in the study. Each graph shown represents a different thickness of UHMW. The parametric study shows clear trends that the thicker the piezoelectric element is in comparison to the UHMW bearing the higher the voltage output.

It is clear from the results of the parametric study that the thickness ratio of the configuration used in the experimental testing is not the optimum configuration. The study predicts that an $8 \mathrm{~mm}$ thick stack generates the highest power output, however, it is important to note that for some resistances a $7 \mathrm{~mm}$ stack will generate a higher voltage output than the 8 $\mathrm{mm}$ stack, so in some cases it may be advantageous from a fatigue perspective to have the extra $.5 \mathrm{~mm}$ thickness of UHMW on the top and bottom of the stack. As shown in Figure 8 the optimum configuration to produce the highest expected power would be a $10 \mathrm{~mm}$ thick disk of UHMW with an $8 \mathrm{~mm}$ thick piezoelectric embedded in the center of the disk. This takes into account an additional parametric study that was conducted concerning the effect the lateral placement of the piezoelectric stack has on the voltage output. The results of this study can be seen in Figure 9. This study shows that locating the piezoelectric stack at the center of the bearing or closest to the edge of the bearing give the highest voltage output. The optimum configuration (an $8 \mathrm{~mm}$ thick piezoelectric in the center of the bearing) gives an average power output of $0.37 \mathrm{~mW}$ per step over its optimal load resistance of $265 \mathrm{k} \Omega$. Given this power output and considering the fact that the eventual design will incorporate four piezoelectric stacks into the UHMW bearing Figure 10 presents the average power output per step expected for the optimal configuration considering four embedded stacks. Considering that the average person takes 9,500 steps a day,$^{18}$ one embedded piezoelectric element would be able to produce $4.76 \mathrm{~J}$ in a day and the total energy output for the device containing four embedded piezoelectrics in a day is $19.04 \mathrm{~J}$.
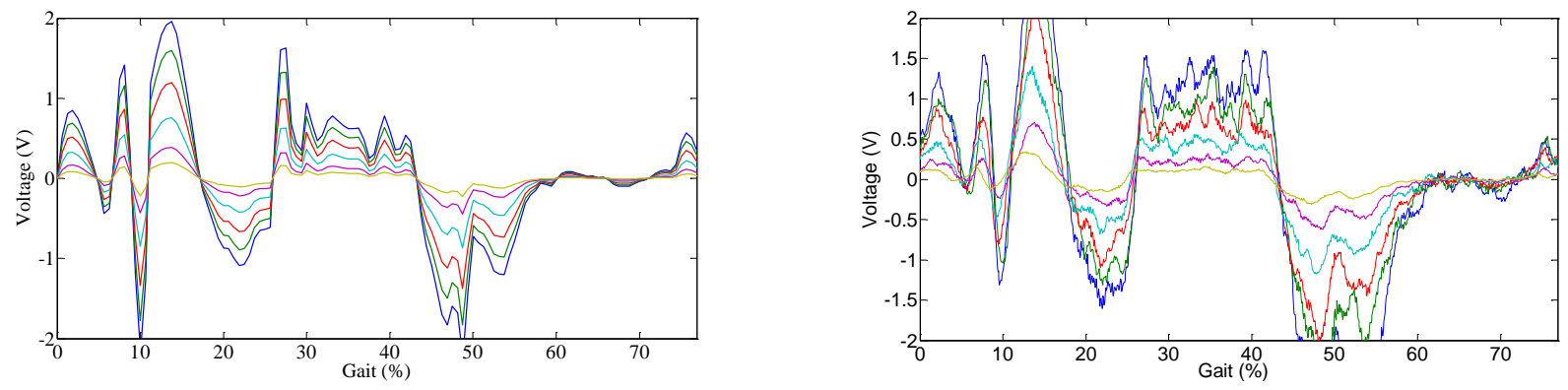

Figure 6. Comparison of simulation results and data collection for a monolithic piezoelectric.
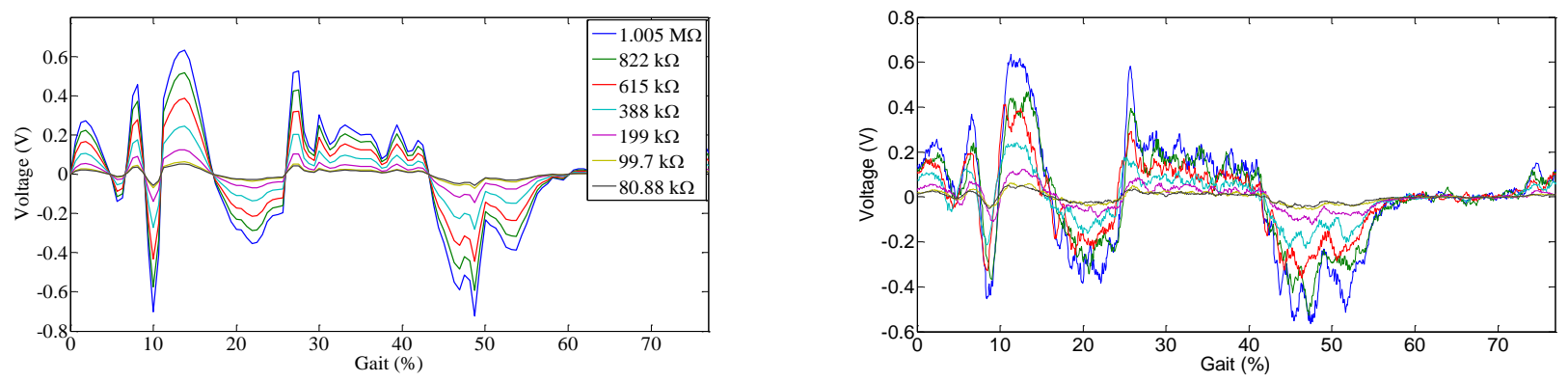

Figure 7. Comparison of simulation results and data collected for a monolithic piezoelectric embedded in UHMW. 
(a)

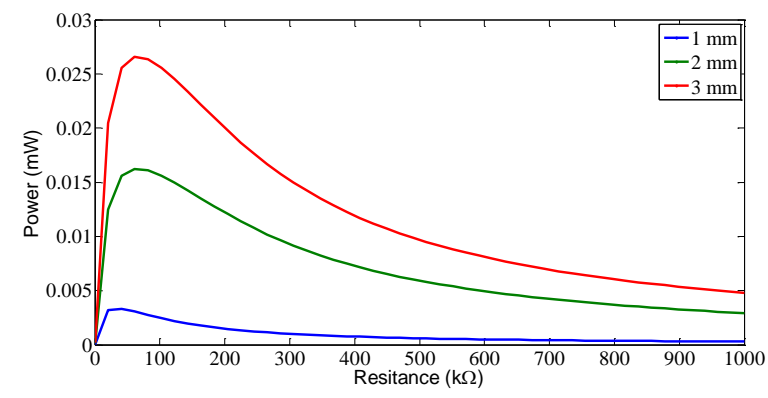

(c)

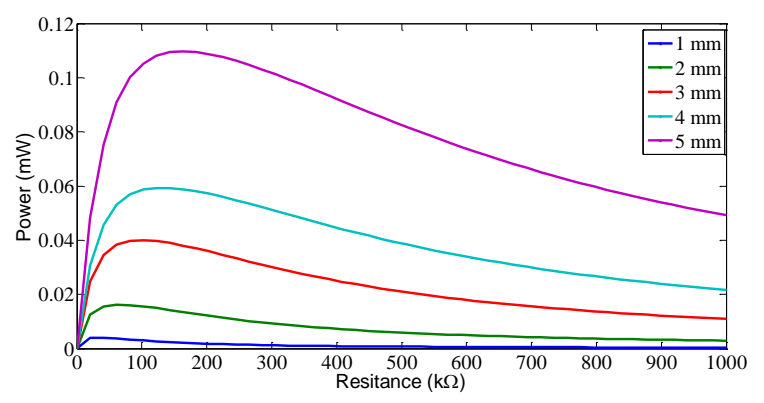

(e)

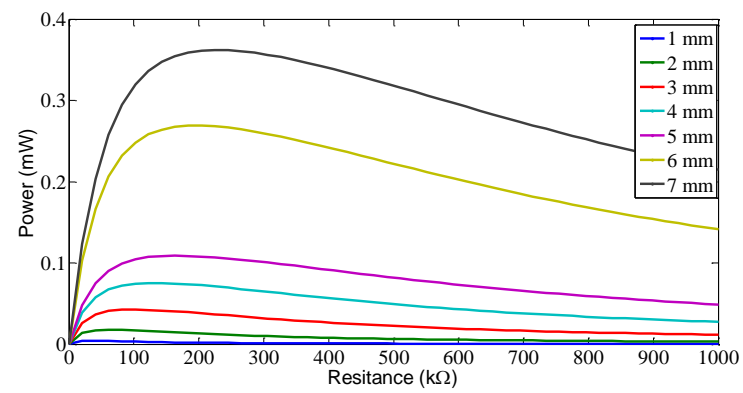

(b)

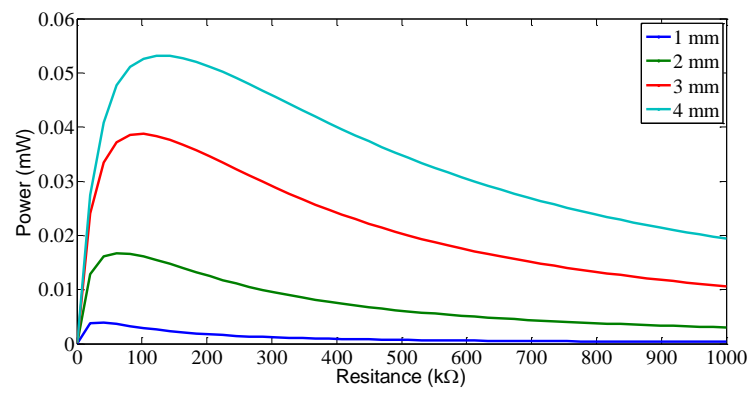

(d)

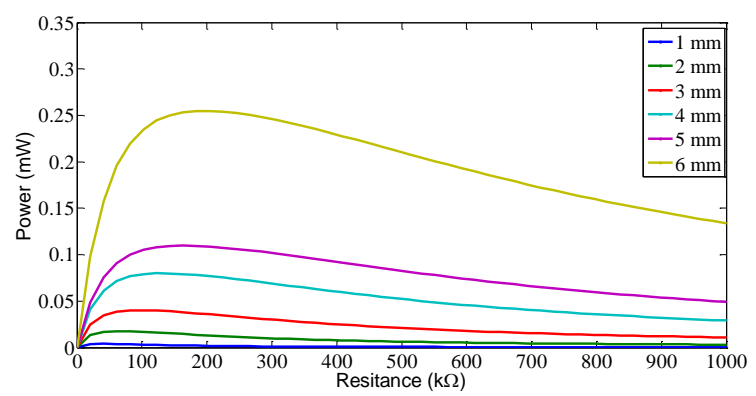

(f)

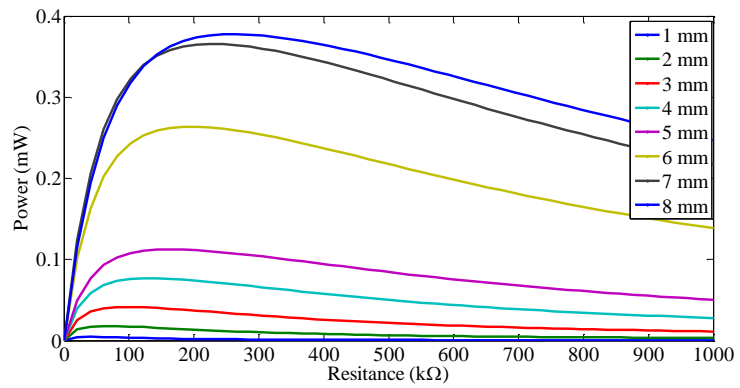

Figure 8. Results of parametric study of the effect of thickness of the piezoelectric element and the UHMW with N=100 for UHMW thicknesses of (a) $5 \mathrm{~mm}$, (b) $6 \mathrm{~mm}$, (c) $7 \mathrm{~mm}$, (d) $8 \mathrm{~mm}$, (e) $9 \mathrm{~mm}$, and (f) $10 \mathrm{~mm}$.

\section{CONCLUSION}

This work explores the use of piezoelectric materials as a power source for low power sensors given the force input of the normal human walking gait. Specifically, it is proposed to embed piezoelectric stacks into the UHMW tibial bearing surface of a total knee replacement unit to sense forces in the knee during surgery and postoperatively, as well as harvest energy to power the sensor. While many studies have investigated in-vivo sensing, few studies have investigated using piezoelectric materials as a power source for both in-vitro and in-vivo data collection. In this study, the open source biomechanical modeling software OpenSim has been used to approximate the knee forces exerted in the human knee for the average sized man. Using uniaxial compression testing, an electromechanical model has been verified so that parametric studies could be conducted in order to ascertain the optimum configuration for the thicknesses of the UHMW and piezoelectric stack. The electromechanical model then calculates the voltage and power output for this optimum case which is an $8 \mathrm{~mm}$ thick, $4 \mathrm{~mm}$ diameter PZT-5H piezoelectric stack with 100 layers embedded in a $10 \mathrm{~mm}$ thick UHMW tibial bearing. Results show that $0.37 \mathrm{~mW}$ of average power can be generated by one stack. The device, having four stacks embedded within it, will be able to produce $19.04 \mathrm{~J}$ of power over the course of one day. These power levels are thought to be sufficient to power a low power sensor for use in in-vitro and in-vivo data collection in TKR patients ${ }^{19}$. 


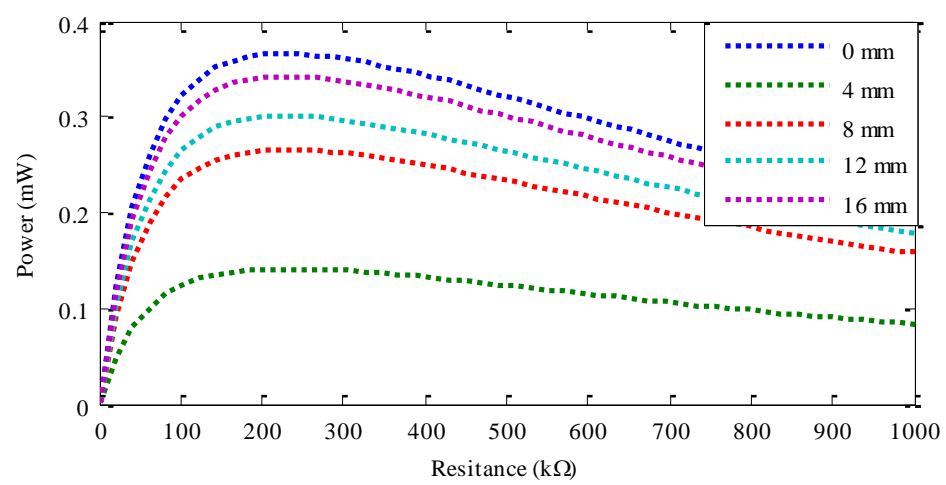

Figure 9. Parametric study results for lateral placement of the piezo $(\mathrm{N}=100)$.

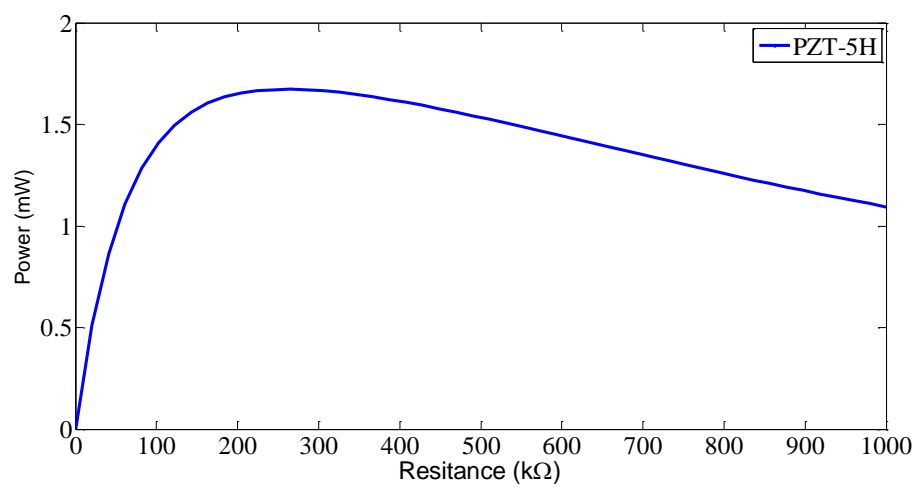

Figure 10. Average power output from optimum configuration (100 layer PZT-5H stack $8 \mathrm{~mm}$ thick).

\section{REFERENCES}

[1] Kaufman, K. R., Kovacevic, N., Irby, S. E. and Colwell, C. W., "Instrumented implant for measuring tibiofemoral forces," J.Biomech. 29(5), 667-671 (1996).

[2] Morris, B. A., D'Lima, D. D., Slamin, J., Kovacevic, N., Arms, S. W., Townsend, C. P. and ColwellJr, C. W. , "e-Knee: Evolution of the Electronic Knee Prosthesis Telemetry Technology Development," the Journal of Bone \& Joint Surgery 83, S62-66 (2001).

[3] Wasielewski, R. C., Galat, D. D. and Komistek, R. D., "Correlation of compartment pressure data from an intraoperative sensing device with postoperative fluoroscopic kinematic results in TKA patients," J.Biomech. 38(2), 333-339 (2005).

[4] D’Lima, D. D., Patil, S., Steklov, N., Chien, S. and Colwell Jr, C. W., "In vivo knee moments and shear after total knee arthroplasty," J.Biomech. 40, S11-S17 (2007).

[5] Kirking, B., Krevolin, J., Townsend, C., Colwell Jr, C. W. and D’Lima, D. D. , "A multiaxial force-sensing implantable tibial prosthesis," J.Biomech. 39(9), 1744-1751 (2006).

[6] D’Lima, D. D., Fregly, B. J., Patil, S., Steklov, N. and Colwell, C. W., "Knee joint forces: prediction, measurement, and significance," Proceedings of the Institution of Mechanical Engineers, Part H: Journal of Engineering in Medicine 226(2), 95-102 (2012).

[7] Wright, T. M., Goodman, S. B. and American Academy of Orthopaedic Surgeons, [Implant Wear in Total Joint Replacement: Clinical and Biologic Issues, Material and Design Considerations: Symposium, Oakbrook, Illinois, October 2000], American Academy of Orthopaedic Surgeons (2001).

[8] Harrington, I. J., "A bioengineering analysis of force actions at the knee in normal and pathological gait," Biomed.Eng. 11(5), 167-172 (1976). 
[9] Kaufman, K., An, K., Litchy, W. and Chao, E., "Physiological prediction of muscle forces-I. Theoretical formulation," Neuroscience 40(3), 781-792 (1991).

[10] Morrison, J., "The mechanics of the knee joint in relation to normal walking," J.Biomech. 3(1), 51-61 (1970).

[11] Paul, J. P., "Force actions transmitted by joints in the human body," Proc.R.Soc.Lond.B.Biol.Sci. 192(1107), 163-172 (1976).

[12] Wilson, B. E., Anton, S. R. and Meneghini, R. M., "Development of Biomechanical Knee Force Model for Evaluation of Piezoelectric Sensors for In-Vivo Monitoring," ASME 2014 Conference on Smart Materials, Adaptive Structures and Intelligent Systems, SMASIS2014-7692 (5 pp.) (2014).

[13] Delp, S. L., Anderson, F. C., Arnold, A. S., Loan, P., Habib, A., John, C. T., Guendelman, E. and Thelen, D. G., "OpenSim: open-source software to create and analyze dynamic simulations of movement," IEEE Transactions on Biomedical Engineering, 54(11), 1940-1950 (2007).

[14] "IEEE Standard on Piezoelectricity," ANSI/IEEE Std 176-1987, (1987).

[15] Kurtz, S. M., Muratoglu, O. K., Evans, M. and Edidin, A. A., "Advances in the processing, sterilization, and crosslinking of ultra-high molecular weight polyethylene for total joint arthroplasty," Biomaterials 20(18), 16591688 (1999).

[16] D'Lima, D., Fregly, B. and Colwell, C., "Implantable sensor technology: measuring bone and joint biomechanics of daily life in vivo," Arthritis Research \& Therapy 15(203), 8 pp. (2013).

[17] Krzypow, D. J. and Rimnac, C. M., "Cyclic steady state stress-strain behavior of UHMW polyethylene," Biomaterials 21(20), 2081-2087 (2000).

[18] Bohannon, R. W., "Number of pedometer-assessed steps taken per day by adults: a descriptive meta-analysis," Phys.Ther. 87(12), 1642-1650 (2007).

[19] Roundy, S., Leland, E. S., Baker, J., Carleton, E., Reilly, E., Lai, E., Otis, B., Rabaey, J. M., Wright, P. K. and Sundararajan, V. , "Improving power output for vibration-based energy scavengers," IEEE Pervasive Computing, 4(1), 28-36 (2005). 